EPJ Web of Conferences 60, 20032 (2013)

DOI: $10.1051 /$ epjconf $/ 20136020032$

C Owned by the authors, published by EDP Sciences, 2013

\title{
Search for direct top squark pair production in final states with one electron or muon using $21 \mathrm{fb}^{-1}$ of ATLAS data
}

\author{
K. Rosbach ${ }^{1, a}$ on behalf of the ATLAS collaboration \\ ${ }^{1}$ Université de Genève, 24, Quai Ernest-Ansermet, CH-1211 Genève 4, Switzerland
}

\begin{abstract}
This article presents latest results of the search for top squark pair production in final states with one isolated lepton, jets, and missing transverse momentum in $\sqrt{s}=8 \mathrm{TeV}$ pp collisions using $\mathrm{L}=21 \mathrm{fb}^{-1}$ of data recorded with the ATLAS detector at the LHC. Two top squark decay scenarios are considered: (a) to a top quark and a long-lived undetected neutral particle (LSP), (b) to a bottom quark and a chargino, where the chargino decays via an on- or off-shell $\mathrm{W}$ boson to the LSP. The analysis also employs a new dedicated shape-fit method to target the challenging parameter region where $m(\tilde{t})$ is close to the kinematic boundary $m(t)+m(L S P)$.
\end{abstract}

\section{Introduction}

Naturalness arguments for weak-scale supersymmetry favour a supersymmetric partner of the top quark (top squark, $\tilde{t}$, or "stop") with a mass close to its Standard Model counterpart.

This article presents a search for directly produced stop pairs, based on a recent conference contribution by the ATLAS collaboration [1]. Two decay channels are considered separately (figure 1). The search uses the full 2012 dataset of pp collisions at $\sqrt{s}=8 \mathrm{TeV}$, recorded with the ATLAS detector [2] at the LHC, with an integrated luminosity of $20.7 \mathrm{fb}^{-1}$.

Left- and right-handed top quark each have a supersymmetric partner, $\tilde{t}_{L}$ and $\tilde{t}_{R}$, which mix to the mass eigenstates $\tilde{t}_{1}$ and $\tilde{t}_{2}$. Only the lighter one, $\tilde{t}_{1}$, is considered here.
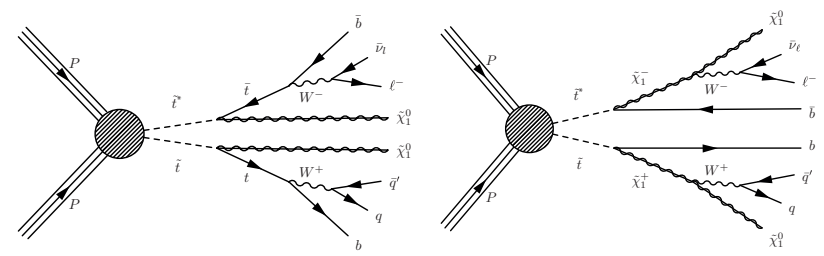

Figure 1. Stop decays targeted by this analysis. The final state objects are the same as in $t \bar{t}$ production, with additional missing momentum from the 2 neutralinos. left: "top+neutralino" ( $\mathrm{tN})$, right: "bottom+chargino" (bC).

\section{Event Selection}

Event kinematic properties differ between the decay modes and also depend on the masses of the SUSY particles. To maximise the sensitivity, six signal regions (SRs) are defined (table 1), sharing a common preselection.
- single lepton or missing transverse momentum trigger,

- one isolated electron or muon with transverse momentum $p_{T}>25 \mathrm{GeV}$, no other $e$ or $\mu$ with $p_{T}>10 \mathrm{GeV}$,

- at least 4 jets with $p_{T}>80,60,40,25 \mathrm{GeV}$, at least one b-tagged (additional requirements for SRbC2 \& 3),

- high missing transverse momentum $\left(E_{\mathrm{T}}^{\mathrm{miss}}\right)$ and missing transverse $\mathrm{W}$ mass $\left(m_{T}(W)\right)$, as detailed in table 1 ,

- the leading two jets are not aligned with $E_{\mathrm{T}}^{\text {miss }}: \Delta \phi>0.8$, in order to reduce the impact of fake $E_{\mathrm{T}}^{\mathrm{miss}}$ resulting from a possible jet energy mismeasurement.

Table 1. Signal region definitions (based on [1]). SRtN1 is the foundation for a shape fit in $E_{\mathrm{T}}^{\text {miss }}$ and $m_{T}(W)$ and applies looser cuts (section 4). Dedicated variables are described in section 5.

\begin{tabular}{lrrr}
\hline & SRtN1 & SRtN2 & SRtN3 \\
\hline$E_{\mathrm{T}}^{\text {miss }}(\mathrm{GeV})$ & 100 & 200 & 275 \\
$E_{\mathrm{T}}^{\mathrm{miss}} / \sqrt{H_{\mathrm{T}}}\left(\mathrm{GeV}^{1 / 2}\right)$ & 5 & 13 & 18 \\
$m_{T}(W)(\mathrm{GeV})$ & 60 & 140 & 200 \\
$a m_{T 2}(\mathrm{GeV})$ & - & 170 & 175 \\
$m_{T 2}^{\tau}(\mathrm{GeV})$ & - & - & 80 \\
$m_{j j j}$ & yes & yes & yes \\
\hline & SRbC1 & SRbC2 & SRbC3 \\
\hline$E_{\mathrm{T}}^{\text {miss }}(\mathrm{GeV})$ & 150 & 160 & 160 \\
$E_{\mathrm{T}}^{\text {miss }} / \sqrt{H_{\mathrm{T}}}(\mathrm{GeV} 1 / 2)$ & 7 & 8 & 8 \\
$m_{T}(W)(\mathrm{GeV})$ & 120 & 120 & 120 \\
am & - & 175 & 200 \\
$m_{T 2}(\mathrm{GeV})$ & - & 550 & 700 \\
isolated track veto & yes & yes & yes \\
Number of b-jets $\geq$ & 1 & 2 & 2 \\
leading b-jet $p_{T}(\mathrm{GeV})$ & 25 & 100 & 120 \\
second b-jet $p_{T}(\mathrm{GeV})$ & - & 50 & 90 \\
\hline
\end{tabular}

\footnotetext{
a e-mail: kilian.rosbach@unige.ch
} 


\section{Background Estimation}

To estimate background contributions from $t \bar{t}$ and $\mathrm{W}+$ jets processes, two control regions (CRs) are defined for each SR: For both the $t \bar{t}$ and $\mathrm{W}+$ jets $\mathrm{CR}$, the $m_{T}(W)$ requirement is lowered to $60-90 \mathrm{GeV}$. For the $\mathrm{W}+$ jets $\mathrm{CR}$, the b-tag requirement is replaced by a b-tag veto. The remaining cuts are kept unchanged or loosened slightly to retain a sufficient number of events in every $\mathrm{CR}$.

Minor backgrounds from $t \bar{t}+\mathrm{V}, \mathrm{VV}$ (diboson) or single top quark events are estimated directly from Monte Carlo (MC) simulations. Contributions from $\mathrm{Z}+$ jets and QCD multijet events are negligible.

The dominant systematic uncertainty in this analysis results from theoretical and modelling uncertainties affecting the transfer factors in the $\mathrm{CR} \rightarrow \mathrm{SR}$ extrapolation. The uncertainties are determined from $\mathrm{MC}$ comparisons and are found to be $7-42 \%$ for the $m_{T}(W)$ extrapolation, and an additional $25 \%$ for the extrapolation from events with no b-jets to events with at least one b-jet.

\section{Shape Fit}

When the mass difference between $\tilde{t}$ and its decay products is small, signal events have similar kinematics properties to $t \bar{t}$ background events.

Region $\mathrm{tN} 1$ targets this challenging part of the mass plane close to the diagonal. Instead of definining signal and control regions, $\mathrm{tN} 1$ is subdivided into $3 \times 5$ bins of and $m_{T}(W)$; some more signal-like, others more backgroundlike, an example is shown in figure 2.

While the statistical treatment is more complex than for the single-bin regions, the additional shape information greatly improves the sensitivity.

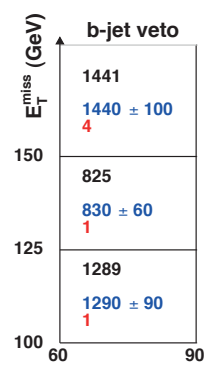

\begin{tabular}{|c|c|c|c|}
\hline \multicolumn{4}{|c|}{ b-jet requirement } \\
\hline 2591 & 663 & 113 & 235 \\
\hline $\begin{array}{l}2590 \pm 100 \\
19\end{array}$ & $\begin{array}{l}700 \pm 150 \\
22\end{array}$ & $\begin{array}{l}101 \pm 26 \\
15\end{array}$ & $\begin{array}{l}262 \pm 34 \\
41\end{array}$ \\
\hline 1962 & 721 & 119 & 165 \\
\hline $\begin{array}{l}1960 \pm 60 \\
10\end{array}$ & $\begin{array}{l}760 \pm 120 \\
13\end{array}$ & ${ }_{8}^{145 \pm 23}$ & $\begin{array}{l}174 \pm 28 \\
18\end{array}$ \\
\hline 3122 & 1521 & 268 & 253 \\
\hline $\begin{array}{l}3120 \pm 120 \\
14\end{array}$ & $\begin{array}{l}1540 \pm 260 \\
16\end{array}$ & $290 \pm 60$ & $\begin{array}{l}250 \pm 60 \\
16\end{array}$ \\
\hline
\end{tabular}

Figure 2. The shape fit uses bins of $E_{\mathrm{T}}^{\text {miss }}$ and $m_{T}(W)$ with different b-jet requirements. Observed counts are shown in black, SM expectations in blue, and a signal benchmark point with $m_{\tilde{t}}=350 \mathrm{GeV}, m_{\chi_{1}^{0}}=150 \mathrm{GeV}$ in red (based on [1]).

\section{Dedicated Variables}

The transverse $\mathrm{W}$ mass combines $E_{\mathrm{T}}^{\mathrm{miss}}$ and lepton $p_{T}$ : $m_{T}(W)=\sqrt{2 p_{T}^{\ell} E_{\mathrm{T}}^{\mathrm{miss}} \cdot(1-\cos (\Delta \varphi))}$. Its distribution has a kinematic endpoint at $m_{W}$ and hints at the presence of an intermediate $\mathrm{W}$ boson.

$a m_{T 2}$ and $m_{T 2}^{\tau}$ are two specialized extensions of the $m_{T}(W)$ variable for events with two invisible particles, as illustrated in figure 3. They are related to the minimal invisible transverse mass compatible with observed kinematics. Examples are shown in figure 4.

Two variables further suppress dileptonic $t \bar{t}$ events: a hadronic top mass cut, based on the invariant mass of 3 jets; and a veto against isolated tracks not assigned to the reconstructed lepton (events with an unidentified lepton or a 1-prong $\tau$ decay).
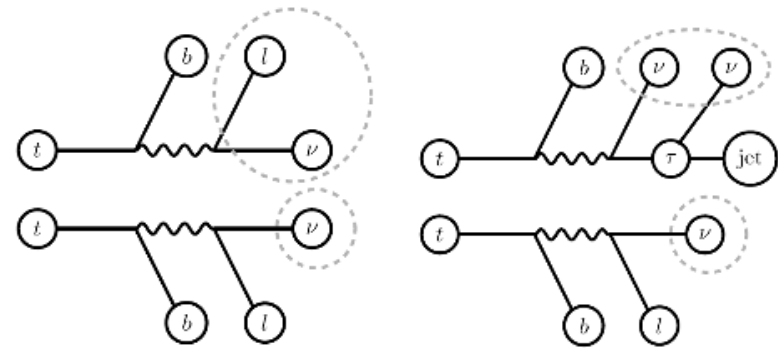

Figure 3. Purpose of $m_{T 2}$ variations. left: partially reconstructed dileptonic $t \bar{t}$ event. $a m_{T 2}$ combines visible objects and invisible masses of $m_{W}$ and $0 \mathrm{GeV}$ to reconstruct 2 tops, right: event with hadronic $\tau$ decay. $m_{T 2}^{\tau}$ uses two invisible masses of $0 \mathrm{GeV}$.
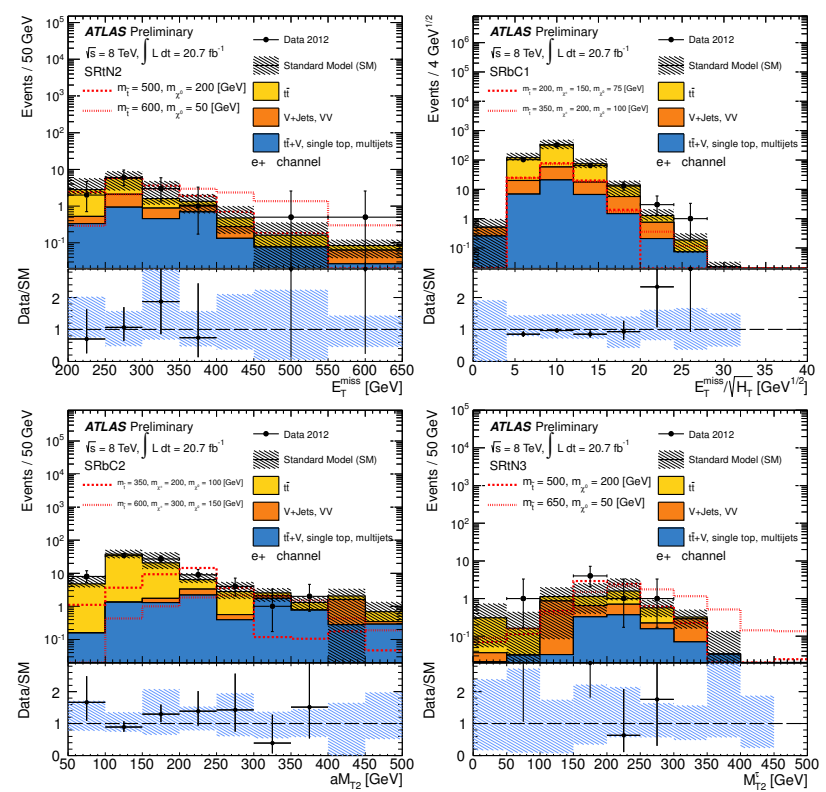

Figure 4. Data/MC comparison of important variables in different signal regions (from [1]). top: $E_{\mathrm{T}}^{\text {miss }}$ and $E_{\mathrm{T}}^{\text {miss }}$ significance, bottom: the $m_{T 2}$ variations $a m_{T 2}$ and $m_{T 2}^{\tau}$.

\section{Results and Statistical Interpretation}

The yields summarized in table 2 are used in a likelihood ratio test based on simultaneous fits of corresponding signal and control regions. It is run in several variations to test the SM-only hypothesis and to compute exclusion limits for the signal processes (figure 5), using the signal region with the best expected sensitivity at each mass point.

Upper limits for a generic BSM model are derived to allow simple exclusion estimates for alternative models 

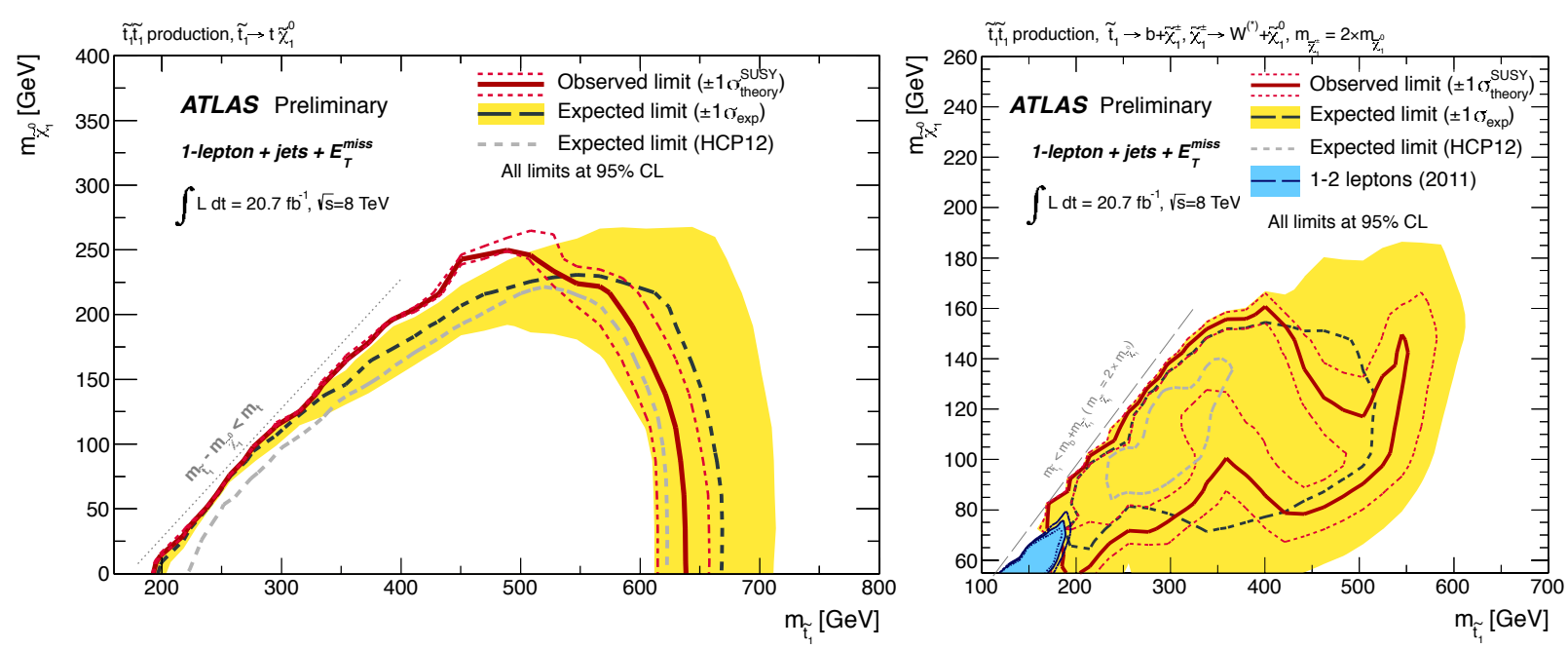

Figure 5. Excluded $\tilde{t}$ and LSP masses at 95\% CL (from [1]). Expected limits are shown in black. Observed limits (in red) are affected by uncertainties on the predicted cross-sections. Results based on $13 \mathrm{fb}^{-1}$ are shown in grey for comparison. Left: $\tilde{t} \rightarrow t+\chi_{1}^{0}$ decay; improvements stem from the shape fit close to the diagonal, and from the $m_{T 2}$ variations at higher $m(\tilde{t})$. Right: $\tilde{t} \rightarrow b+\chi_{1}^{ \pm}$decay (with $\chi_{1}^{ \pm} \rightarrow \chi_{1}^{0}+W$ and $\left.m\left(\chi_{1}^{ \pm}\right)=2 \times m\left(\chi_{1}^{0}\right)\right)$; exclusion at higher $m(\tilde{t})$ is improved by adding new SRs with tighter b-jet requirements.

Table 2. Expected and observed event yields (based on [1]). Masses are given in $\mathrm{GeV}$. For the shape fit region $\mathrm{tN} 1$, the sum of bins with a b-jet requirement is shown.

\begin{tabular}{|c|c|c|c|c|c|}
\hline \multicolumn{3}{|c|}{$m(\tilde{t}) \quad m\left(\chi_{1}^{0}\right)$} & SRtN1 & SRtN2 & SRtN3 \\
\hline 225 & \multicolumn{2}{|l|}{25} & $1075 \pm 26$ & $2.2 \pm 1.0$ & $0.9 \pm 0.6$ \\
\hline 350 & \multicolumn{2}{|l|}{150} & $201 \pm \quad 5$ & $2.0 \pm 0.5$ & $0.5 \pm 0.3$ \\
\hline 500 & \multicolumn{2}{|l|}{200} & $71.9 \pm 1.4$ & $14.9 \pm 0.6$ & $6.8 \pm 0.4$ \\
\hline 600 & \multicolumn{2}{|l|}{50} & $30.2 \pm 0.5$ & $13.9 \pm 0.4$ & $11.6 \pm 0.3$ \\
\hline \multicolumn{3}{|c|}{ SM expectation } & $11833 \pm 378$ & $13 \pm 3$ & $5 \pm 2$ \\
\hline \multicolumn{3}{|c|}{ Data observed } & 11733 & 14 & 7 \\
\hline \multicolumn{3}{|c|}{$m(\tilde{t}) m\left(\chi_{1}^{ \pm}\right) m\left(\chi_{1}^{0}\right)$} & SRbC1 & SRbC2 & SRbC3 \\
\hline 200 & 150 & 75 & $233 \pm 18$ & $8 \pm 4$ & $3.1 \pm 3.1$ \\
\hline 350 & 200 & 100 & $166 \pm 5$ & $38.3 \pm 2.5$ & $11.1 \pm 1.4$ \\
\hline 600 & 300 & 150 & $26.3 \pm 0.6$ & $12.5 \pm 0.4$ & $9.0 \pm 0.4$ \\
\hline \multicolumn{3}{|c|}{ SM expectation } & $482 \pm 76$ & $18 \pm 5$ & $7 \pm \quad 3$ \\
\hline \multicolumn{3}{|c|}{ Data observed } & 456 & 25 & 6 \\
\hline
\end{tabular}

(table 8 in [1]). They can be used as long as the expected signal contamination in the CRs is low. The effect of different assumptions on the stop mixing and the resulting top polarization in stop decays is illustrated in figure 6 for a fixed LSP mass of $50 \mathrm{GeV}$.

\section{Summary}

A search for stop quarks using the full dataset of 2012 ATLAS pp collisions was performed. No significant excesses were observed in the event yields. Using additional variables and a more sophisticated fit technique, the previously derived exclusion limits could be pushed closer to the diagonal in the $\tilde{t}$-LSP mass plane and toward higher $\tilde{t}$ masses.

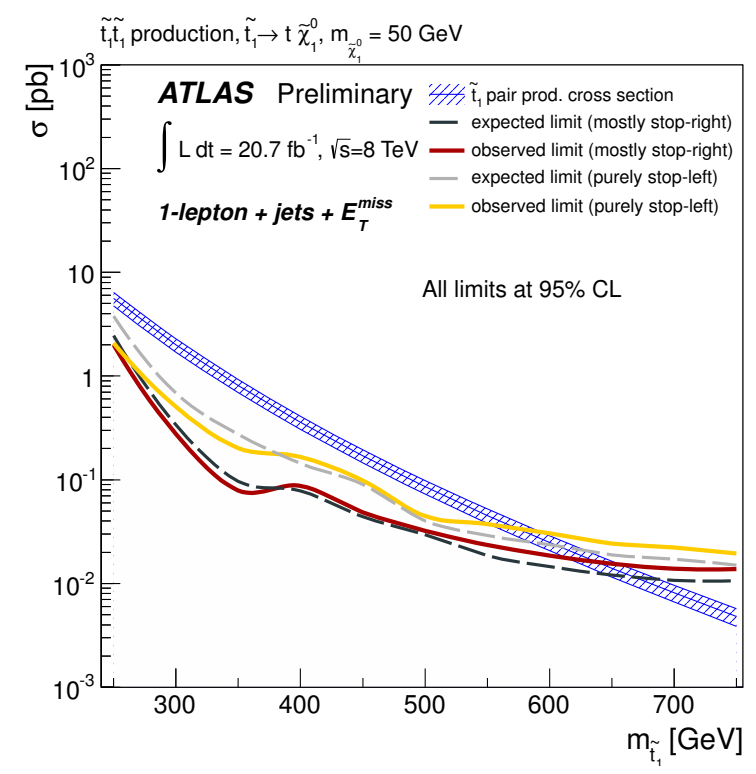

Figure 6. Impact of $\tilde{t}_{1}$ mixing for a fixed neutralino mass of $50 \mathrm{GeV}$ (from [1]). A mostly $(\sim 70 \%) \tilde{t}_{R}$ model is used throughout the analysis (red line). A purely $\tilde{t}_{L}$ model assumption reduces the exclusion reach by about $75 \mathrm{GeV}$ (yellow line).

\section{References}

[1] ATLAS collaboration, Search for direct top squark pair production in final states with one isolated lepton, jets, and missing transverse momentum in $\sqrt{s}=8 \mathrm{TeV}$ pp collisions using $21 \mathrm{fb}^{-1}$ of ATLAS data (2013), ATLAS-CONF-2013-037, https : //cds . cern. ch/record/1532431

[2] ATLAS collaboration, Journal of Instrumentation $\mathbf{3}$, S08003 (2008) 